\title{
Connections between MWG Star Clusters and Dwarf Galaxies
}

\author{
Kim A. Venn \\ University of Victoria, \\ PO Box 3055 STN CSC, Victoria, BC, Canada V8W 3P6 \\ email: kvenn@uvic.ca
}

\section{Introduction}

It seems that in the past decade, there have been two paradigm shifts regarding star clusters. Firstly, the observational evidence for multiple stellar populations requires more extended and often complex star formation histories in star clusters. Secondly, theoretical models that form globular clusters in dwarf galaxies that are accreted at very early epochs $(\mathrm{z}>5)$ are able to reproduce the age-metallicity relations observed. For the accretion scenario to be viable, globular clusters should also resemble the chemistry of at least some dwarf galaxies.

\section{A Chemical Comparison}

We investigate the chemical connections between star clusters and dwarf galaxies here, but sadly, this is a short examination. At low metallicities, the chemical composition of globular clusters, Galactic halo stars, and dwarf galaxies are all similar (Venn et al. 2004, Tafelmeyer et al. 2010, Frebel \& Norris 2011), with few clues as to potential differences in their origins or birth places. Only at intermediate metallicities $([\mathrm{Fe} / \mathrm{H}]>-1.0)$ do dwarf galaxies show unique chemical properties due to their slower star formation histories compared to most stars in the Galaxy, and nearly all of those are associated with the Sgr dwarf remnant. A few interesting exceptions include Rup 106 (see talk by Doug Geisler) and Pal 1 (Sakari et al. 2011), both showing unique chemical signatures but neither associated with the Sgr remnant.

When chemical abundances differ in dwarf galaxies, this can usually be traced through several elements, and not only the $[\alpha / \mathrm{Fe}]$ ratios. For example, Pal 12 and Ter 7 both show lower abundances of $[\mathrm{Na} / \mathrm{Fe}],[\mathrm{Al} / \mathrm{Fe}],[\mathrm{Cu} / \mathrm{Fe}]$, and $[\mathrm{Zn} / \mathrm{Fe}]$ suggestive of metallicity dependent AGB and/or SNe Ia yields These lower abundances are also seen in dwarf galaxies, such as low $\mathrm{Na}$ in Fornax (Letarte et al. 2010), low $\mathrm{Cu}$ in the LMC (Pompeia et al. 2008), and all four are low in the Sgr field stars (Monaco et al. 2007)

\section{Younger Clusters From Dwarf Galaxies?}

A "vital diagram" of mass versus half light radius for Sgr clusters suggests that some open clusters could be associated with the Sgr dwarf remnant (Law \& Majewski 2010). These include the peculiar young halo clusters, such as Whiting 1, and open clusters Berkeley 29 and Saurer 1. The ages ( $\sim$ Gyr) and metallicities $([\mathrm{Fe} / \mathrm{H}] \sim-0.6)$ of these clusters are similar to the Sgr clusters Pal 12 and Ter 7, however the chemistries of Be 29 and $\mathrm{Sa} 1$ such as $[\alpha / \mathrm{Fe}]$ and $[\mathrm{Na} / \mathrm{Fe}]$ ratios are more similar to stars in the Galaxy (Yong et al. 2005, Carraro et al. 2004; see Figure 1). Upper limits on their $\mathrm{Cu}$ and $\mathrm{Zn}$ 
abundances do not add further constraints. Heavier elements such as La and Ba are inconclusive.

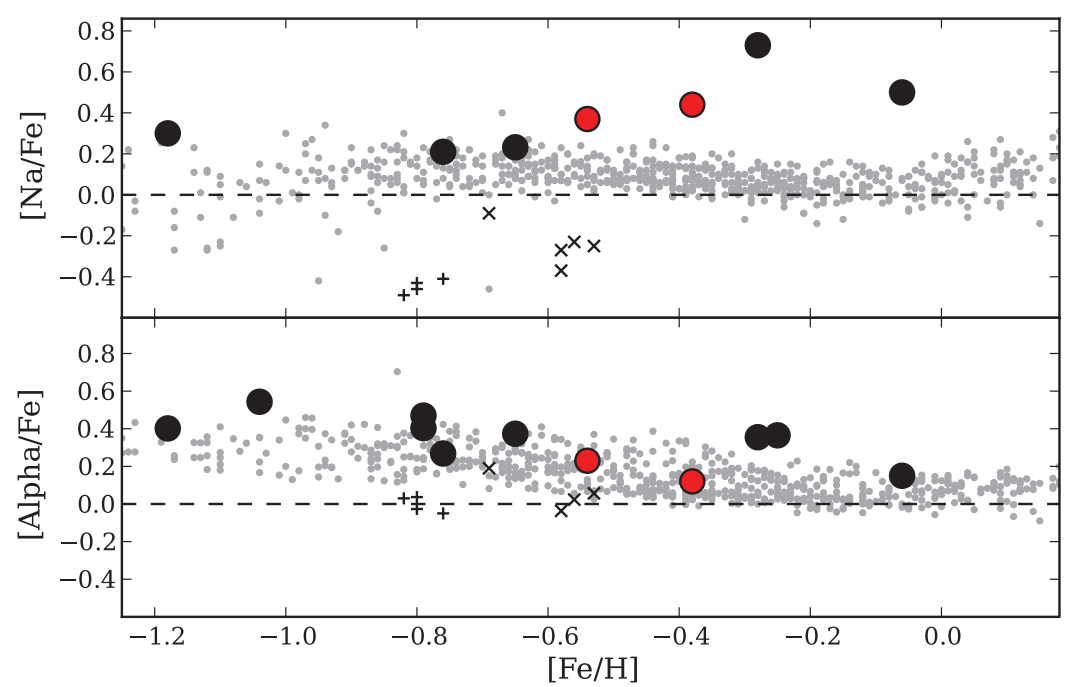

Figure 1. Elemental abundances for Berkeley 29 and Saurer 1 (red points; Yong et al. 2005, Carraro et al. 2004), and other Galactic globular clusters (black points, Pritzl et al. 2005) and Galactic field stars (grey points, Venn et al. 2004). The Sgr clusters Pal 12 and Ter 7 are shown by crosses and " $\mathrm{x}$ " marks (Cohen 2004, Sbordone et al. 2007), respectively, and are clearly lower than the Galactic stars including Be 29 and Sa 1. [Alpha/Fe] is an average of Mg and Ca.

\section{Discussion}

The similarities in age and metallicity of young stellar clusters in the Galactic halo with Sgr dwarf remnant clusters is striking, and seems unlikely to be only a coincidence. Perhaps these clusters are associated with the Sgr merger through gas deposition and compressed along the orbital plane (e.g., K. Bekki's talk). Or perhaps the young stellar clusters are associated with the Galactic disk, and their ages are similar to the Sgr clusters if they were triggered to collapse through tidal interactions during the accretion of Sgr. Thus, currently, there are no chemical abundances that link young stellar clusters near the Galactic disk with the Sgr stellar clusters. However, with higher order element abundances (Ba, Sr, Cu, Zn) and homogenized analyses (i.e., see comments by Friel et al. 2010), the origins of the MWG star clusters can be further clarified.

\section{References}

Carraro G., Bresolin F., Villanova S., et al. 2004, AJ, 128, 1676

Cohen J. 2004, AJ, 127, 1545

Friel E., Jacobson H. R., \& Pilachowski C. A. 2010, AJ, 139, 1942

Frebel A. \& Norris J. E. 2011, to appear in Planets, Stars and Stellar Systems Springer (arXiv:1102.1748)

Law D. \& Majewski S. 2010, ApJ, 718, 1128

Letarte B., Hill V., Tolstoy E., et al. 2010, A\&A, 523, 17

Monaco L., Bellazzini M., et al. 2007, A\& $A, 464,201$

Pompeia L., Hill V., Spite M., et al. 2008, A\&A, 480, 379

Pritzl B. J., Venn K. A., \& Irwin M. J. 2005, AJ, 130, 2140 
Sakari C. M., Venn K. A., et al. 2011, ApJ, 740, 106

Sbordone L., Bonifacio P., et al. 2007, A\&BA, 465, 815

Tafelmeyer M., Jablonka P., Hill V., et al. 2010, A\&A, 524, 58

Tolstoy, E., Hill V. \& Tosi, M. 2009, ARAA, 47, 371

Venn K. A., Irwin M. J., Shetrone M. D. et al. 2004, AJ, 128, 1177

Yong D., Carney B. W., \& Teixera de Almeida M. L. 2005, AJ, 130, 597 